\title{
Quadratic Corrections to Harmonic Vibrational Frequencies Outperform Linear Models
}

\author{
Marat Sibaev and Deborah L. Crittenden* \\ Department of Chemistry, University of Canterbury, Christchurch, New Zealand \\ E-mail: deborah.crittenden@canterbury.ac.nz
}

\begin{abstract}
Simulating accurate infrared spectra is a longstanding problem in computational quantum chemistry. Linearly scaling harmonic frequencies to better match experimental data is a popular way of approximating anharmonic effects while simultaneously attempting to account for deficiencies in ab initio method and/or basis set. As this approach is empirical, it is also non-variational and unbounded, so it is important to separate and quantify errors as robustly as possible. Eliminating the confounding factor of methodological incompleteness enables us to explore the intrinsic accuracy of the scaling approach alone. We find that single-coefficient linear scaling methods systematically overcorrect low frequencies, while generally undercorrecting higher frequencies. A two-parameter polynomial model gives significantly better predictions without systematic bias in any spectral region, while a single-parameter quadratic scaling model is parameterized to minimize overcorrection errors while only slightly decreasing predictive power.
\end{abstract}

\footnotetext{
*To whom correspondence should be addressed
} 


\section{Introduction}

It is widely reported ${ }^{1-7}$ and commonly accepted in the scientific literature that $a b$ initio derived harmonic vibrational frequencies tend to be larger than experimentally observed fundamentals, due to the combined effects of anharmonicity and methodological incompleteness. Assuming that these effects are uniform across the spectral range motivates the use of parameterized scaling factors to improve the agreement between predicted and observed fundamental frequencies. ${ }^{1-7}$ Within this empirical framework, separate scaling factors are required for different $a b$ initio method and basis set combinations. ${ }^{1-7}$

However, there are two lines of evidence in the literature to suggest that straightforward linear scaling of normal coordinate force constants may not always be justified.

Firstly, internal coordinate force constant scaling approaches ${ }^{8}$ achieve higher accuracies than simple normal coordinate frequency scaling. However, this comes at the cost of having to define appropriate internal coordinate sets and parameterize different scaling factors for each internal coordinate. Although somewhat lacking in generality, this approach nonetheless illustrates the importance of different scaling factors for different types of molecular motion.

Even within the literature on normal coordinate force constant scaling, there are strong indications that anharmonicity and methodological incompleteness effects are not, in fact, constant across the entire spectral range, with different scaling factors required in low and high frequency regimes. ${ }^{5,6}$ Down-scaling is always recommended for high frequencies, but scaling factors for low frequencies vary significantly in both magnitude and direction across different levels of theory, with a median recommended value around 1.0, corresponding to no scaling correction.

These observations raise a number of questions:

- Can an alternative relationship between anharmonicity and harmonic normal mode frequency be empirically established? 
- Could this underpin a more accurate and/or robust anharmonic correction model that retains the simplicity and generality of a frequency scaling approach?

- Are the low frequency scaling factors primarily accounting for methodological incompleteness rather than anharmonicity?

\section{Methods}

To eliminate the confounding effects of methodological incompleteness, we use the PyPES library of high quality semi-global potential energy surfaces (PES). ${ }^{9}$ This enables us to obtain benchmark anharmonic vibrational frequencies and their harmonic counterparts for 226 unique fundamental vibrational modes. Although reference anharmonic vibrational frequencies are available in the literature for the PES contained within the PyPES library, benchmark harmonic frequencies calculated from these surfaces have not all been available until now. The 50 molecules within the PyPES library vary in size from three to six atoms, and contain a range of different atom types, bonding patterns and molecular topologies. Hence, the vibrational modes of these molecules are expected to form a representative set. All benchmark anharmonic and harmonic frequencies used in this work are provided as Supporting Information.

For larger molecules, where high level ab initio calculations to obtain accurate potential energy surfaces are not feasible, previous work ${ }^{5,6,12}$ suggests that density functionals incorporating around 20\% Hartree-Fock exchange reliably recover harmonic frequencies comparable to those obtained at much higher levels of theory. We therefore benchmark the ability of the B3LYP,${ }^{13}$ B3PW91, ${ }^{14} \mathrm{PBE} 0,{ }^{15} \mathrm{EDF} 2,{ }^{12} \mathrm{M} 05^{16}$ and $\mathrm{M} 06{ }^{17}$ functionals to recover benchmark harmonic frequencies for all molecules in the PyPES library. We also assess the ability of the quadratic correction model defined above to predict anharmonic frequencies from DFT harmonic frequencies.

All calculations are carried out in the atomic orbital basis sets that were used for parameterizing each functional, ${ }^{18-22}$ augmenting each basis set with diffuse functions for 
anionic molecules if not already included. ${ }^{23,24}$ The CRENBL ECP basis ${ }^{25,26}$ is used for atoms larger than Kr. All (TD-)DFT geometry optimizations and subsequent frequency calculations are carried out using the $Q$-Chem 4.2 program package, ${ }^{27}$ employing a EulerMaclaurin - Lebedev product quadrature grid comprising 75 radial points and 302 angular points per radial point, with an SCF convergence threshold of $10^{-8}$ and geometry optimization thresholds decreased by an order of magnitude from their default values.

\section{Results and Discussion}

\section{Anharmonicity Model}

For clarity and consistency, we recast the scale factor approach of Radom et al. ${ }^{5,6}$ (1) as a linear correction model (2).

$$
\begin{aligned}
& \nu \approx \lambda \nu_{\mathrm{e}} \\
& \nu \approx\left(1-c_{1}\right) \nu_{\mathrm{e}}
\end{aligned}
$$

in which $\nu$ represents the benchmark anharmonic frequency we wish to approximate and $\nu_{\mathrm{e}}$ its harmonic equivalent. This enables us to recast the problem of minimizing the difference between scaled and benchmark frequencies as a problem of approximating anharmonicities as a function of harmonic frequencies:

$$
\nu_{\mathrm{e}}-\nu \approx c_{1} \nu_{\mathrm{e}}
$$

Or, equivalently,

$$
\Delta_{\mathrm{anh}} \approx c_{1} \nu_{\mathrm{e}}
$$

The optimal coefficient, $c_{1}$, is determined by least-squares fitting to experimentally derived or benchmark anharmonicities, i.e. by linear regression with $\Delta_{\text {anh }}$ as the response variable and $\nu_{\mathrm{e}}$ as the independent variable, as illustrated in Figure 1(a). As per equa- 
tions (1) and (2), the coefficient, $c_{1}$, derived in this manner is the complement of the scaling factor, $\lambda$, defined by Radom et al. The quality of the model is more evident upon examining the residual differences between predicted and benchmark frequencies:

$$
\Delta_{\text {res }}=\nu_{\text {predicted }}-\nu
$$

shown in Figure 1(b), noting that if no correction is applied to the harmonic frequencies then $\nu_{\text {predicted }}=\nu_{\mathrm{e}}$ and $\Delta_{\text {res }}=\Delta_{\text {anh }}$.

From Figures 1(a) and 1(b), it is clear that a single parameter linear correction model significantly overestimates anharmonicity corrections in the low frequency, low anharmonicity regime while simultaneously underestimating anharmonicity corrections at higher frequencies. Indeed, low frequencies are often overcorrected to such an extent that the 'improved' frequency estimates are, in fact, further from the experimental values than the original harmonic estimates. Points below the red anti-diagonal line on Figure 1(b) fall into this category. Ideally, the trend line on Figure 1(a) would provide a closer fit to the benchmark anharmonicity data, resulting in residual errors in Figure 1(b) narrowly and randomly clustered around the dashed horizontal line.

The tendency of the single parameter linear scaling model to overcorrect low frequency modes is also reflected in the summary statistics presented in Table 1. Although the mean absolute error decreases from 47.0 to $19.6 \mathrm{~cm}^{-1}$, the average error associated with underpredicted frequencies increases from 8.2 to $18.2 \mathrm{~cm}^{-1}$, with corresponding maximum error increasing from 17.3 to $44.0 \mathrm{~cm}^{-1}$. The $c_{1}$ value of 0.039640 corresponds to a $\lambda$ value of 0.96036 , in very good agreement with existing scale factors parameterized for high level correlated ab initio methods across a larger data set; 0.9639 for $\operatorname{CCSD}(\mathrm{T}) / 6$ $311+\mathrm{G}(\mathrm{d}, \mathrm{p}) .{ }^{6}$

It is now evident that the dual scaling factor recommendation of Radom et al. ${ }^{5,6}$ implies that at least a bi-linear model is required to describe trends in anharmonicity as a function of harmonic frequency. However, they do not give an exact prescription for mapping 
scale factor to frequency range. To complete the specification of their model, further optimization to determine the optimal 'cross-over' point would be required.

Given the relatively straightforward relationship between anharmonicity and harmonic frequency apparent upon visual inspection of Figure 1(a), this approach seems needlessly complicated. Instead, we propose a second-order polynomial model:

$$
\Delta_{\mathrm{anh}} \approx c_{1} \nu_{\mathrm{e}}+c_{2} \nu_{\mathrm{e}}^{2}
$$

This produces a much closer fit to the anharmonicity data, as illustrated in Figure 1(c) and summarized in Table 1. Although the polynomial model produces universally more accurate estimates of the benchmark frequencies than the single-parameter linear model, there remains a cluster of outliers in the low frequency, high anharmonicity region, a single outlier at $1052 \mathrm{~cm}^{-1}$ and $\Delta_{\text {anh }}=99 \mathrm{~cm}^{-1}$, and another outlying pair of modes with $\Delta_{\text {anh }}<<0$.

Modes with anomalously high anharmonicities all represent cases in which the assumption of low amplitude vibrations about a single minimum on a PES expanded in normal coordinates breaks down; for low barrier torsional modes (the low frequency, high anharmonicity cluster) and the $\mathrm{NH}_{3}$ inversion mode (the lone outlier at $1052 \mathrm{~cm}^{-1}$ ). In these cases, correcting for anharmonicity by scaling normal coordinate force constants is inappropriate, as internal-coordinate based approaches for expanding the PES and solving the nuclear vibrational Schrödinger equation are required.

The two cases in which anharmonicity increases the fundamental frequencies correspond to antisymmetric stretching modes of excited state $\mathrm{ClO}_{2}$ and $\mathrm{BrO}_{2}$. Early studies attributed this behaviour to $C_{s}$-distortion of the equilibrium geometry producing a very shallow double minimum in the potential. ${ }^{10}$ However, more extensive recent work has concluded that the negative (according to the sign convention adopted here) anharmonicity corrections arise from strong anharmonic coupling between symmetric and asymmetric stretching modes. ${ }^{11}$ Again, a normal coordinate force constant scaling approach is ill- 
suited to capturing these effects.

This is reflected in the residual error data illustrated in Figure 1(d). The polynomial scaling model fails to allow harmonic frequencies to increase toward their anharmonic counterparts, resulting in residual errors as large as, or even worse than, the original harmonic estimates, i.e. $\left|\Delta_{\text {res }}\right|>\left|\Delta_{\text {anh }}\right|$ when $\Delta_{\text {anh }}<0$. In these cases, the 'least worst' prediction would be no change from harmonic.

Otherwise, excluding torsional and inversion mode outliers, residual errors tend to be randomly and narrowly scattered about $\Delta_{\text {res }}=0$. Although the polynomial model significantly outperforms the linear model on this metric, it has the disadvantage of requiring an additional empirical parameter. Further, there remain a number of points below the red anti-diagonal line on Figure 1(d), indicating that although the magnitude and extent of 'worse-than-harmonic' overcorrection errors have decreased, they have not been completely eliminated.

Therefore, we seek a model that; minimizes overcorrection errors rather than minimizing the overall error, requires only a single parameter, and outperforms the single-parameter linear model in every metric. This combination of constraints yields the quadratic model illustrated in Figures 1(e) and 1(f), with $c_{2}=0.00001215$. A major advantage of this model is that it provides a lower bounded estimate of the anharmonicity in most cases. In other words, it generally corrects harmonic frequencies down toward but not beyond their experimental values. This is particularly important when error direction is as important, if not more important, than error magnitude.

For example, corrected frequencies that are higher than their true values will yield lower bounds for derived thermochemical parameters such as enthalpies and entropies. Further, this leads to lower total errors in calculated thermochemical parameters, as anharmonic frequencies that are too high result in smaller errors than frequencies that are too low by the same amount, due to the inverse exponential ansatz.

There remain a handful of cases in which the quadratic model overpredicts the anhar- 
monicity correction, but in each of these cases, the error is small. Overcorrection errors are less than $12 \mathrm{~cm}^{-1}$ in all cases, averaging $3.0 \mathrm{~cm}^{-1}$. Like the polynomial model, the quadratic model fails to account for the rare cases in which the true frequencies are higher than the harmonic frequencies and a negative anharmonicity correction is required. In these cases, the quadratic model does not significantly compound this error, but instead returns frequencies similar within $3 \mathrm{~cm}^{-1}$ of the original harmonic frequencies.

\section{DFT Frequencies}

Errors in (TD-)DFT harmonic frequencies are calculated with reference to benchmark values:

$$
\Delta_{\text {harm }}=\nu_{\mathrm{e}}^{\mathrm{DFT}}-\nu_{\mathrm{e}}
$$

Mean and maximum absolute and signed errors in DFT harmonic frequencies are reported in Table 2. Excited states and molecules containing atoms larger than Kr are excluded from statistical analysis, because using TD-DFT or effective core potentials introduces additional approximations beyond those inherent in the parameterization of each functional, which may further decrease the accuracy of the calculated $\nu_{\mathrm{e}}^{\mathrm{DFT}}$. For completeness, the full set of results is provided as Supporting Information.

The data presented in Table 2 are broadly consistent with previous studies that report mean or RMSD errors in harmonic frequencies of $30-40 \mathrm{~cm}^{-1}$ using B3LYP ${ }^{28}$ and PBE0 ${ }^{28,29}$ with triple zeta basis sets. The minor discrepancy between the literature results and those reported here arises from our use of a larger and more representative test set of molecules and our choice to use the basis sets in which each functional was parameterized.

Of the functionals investigated here, EDF2 is generally the most accurate. This is to be expected, as it was explicitly parameterized to recover CCSD(T)/cc-pVTZ harmonic frequencies. ${ }^{12}$ Nonetheless, significant errors in harmonic frequencies are observed, with a mean absolute deviation of $21.1 \mathrm{~cm}^{-1}$ and maximum absolute error of $82.9 \mathrm{~cm}^{-1}$. Statistically, errors are randomly distributed across the data set. However, upon visual 
inspection of Figure 2(a), it is clear that EDF2 systematically underestimates the frequencies of highly anharmonic modes.

Errors in quadratically corrected DFT-derived anharmonic frequencies are presented in Table 3. Comparing Tables 2 and 3 reveals a strong correlation between mean absolute and maximum errors in DFT harmonic frequencies, and corresponding errors in DFTderived anharmonic frequencies. This implies that residual errors in predicted anharmonic frequencies derive primarily from the inaccuracy of the DFT harmonic frequencies rather than inadequacy of the anharmonicity correction model. This observation is supported by existing literature results, in which anharmonic corrections are calculated using vibrational perturbation theory. Even using this significantly more time consuming and rigorous procedure to account for anharmonicity, errors in calculated anharmonic frequencies are strongly correlated with errors in the underlying harmonic frequencies. ${ }^{29}$

For low frequency modes, the quadratic model predicts only small anharmonicity corrections by construction, and therefore errors in DFT harmonic frequencies translate almost directly into residual errors in predicted anharmonic frequencies, as anticipated above. This behaviour is evident comparing the low anharmonicity regions of Figures 2(a) and $2(b)$.

For high frequency modes, the predicted anharmonic frequencies are scattered randomly about $\Delta_{\text {res }}=0$, as shown in Figure 2(b). This is a consequence of error cancellation, with the quadratic correction model systematically overestimating anharmonic frequencies as it was designed to do, and the EDF2 functional systematically underestimating harmonic frequencies. Although it would be possible to reparameterize the quadratic correction model to reinstate the upper bound behaviour for high frequencies, or further optimize it to achieve maximum error cancellation, we consider it preferable to control for anharmonicity and methodological errors separately so we do not pursue this approach. 


\section{Conclusions}

Overall, we recommend using the quadratic correction model in conjunction with high level ab initio harmonic frequencies, due to its simplicity, accuracy and ability to provide semi-bounded lower estimates of anharmonicities. This approach recovers anharmonic frequencies within $\sim 13 \mathrm{~cm}^{-1}$ of benchmark values, on average, across a diverse range of chemical species. We note that low barrier torsional and inversion modes should be excluded a priori due to the inappropriateness of normal modes for describing these types of motion.

Where high level $a b$ initio harmonic frequency calculations are not feasible, quadratically corrected DFT frequencies reasonably approximate anharmonic stretching frequencies, with mean absolute errors in the $20-30 \mathrm{~cm}^{-1}$ range. However, DFT-derived estimates of anharmonic frequencies are less reliable for lower frequency torsional and bending modes, due to these regions of the potential energy surface being poorly described by DFT methods. In these cases, errors in anharmonic fundamental frequencies predicted by both simple empirical correction models and more rigorous nuclear vibrational structure theories (VPT2) are both dominated by relatively large errors in the DFT harmonic frequencies.

Supporting Information Available: Benchmark data - anharmonic frequencies, harmonic frequencies, anharmonicities; Ab initio data - DFT harmonic frequencies; Statistical model data - predicted anharmonic frequencies. 
Table 1: Mean unsigned, mean signed and maximum signed errors in predicted anharmonic frequencies $\left(\Delta_{\text {res }}\right)$, according to models defined by equation 6 and the parameters given. All values reported in units of $\mathrm{cm}^{-1}$.

\begin{tabular}{|c|c|c|c|c|c|c|c|}
\hline \multirow[b]{2}{*}{ approx } & \multirow[b]{2}{*}{$c_{1}$} & \multirow[b]{2}{*}{$c_{2}$} & \multicolumn{3}{|c|}{$\left\langle\left|\Delta_{\text {res }}\right|\right\rangle$} & \multicolumn{2}{|c|}{$\left|\Delta_{\text {res }}\right|_{\text {max }}$} \\
\hline & & & all & $\begin{array}{c}\nu_{\text {model }} \\
>\nu\end{array}$ & $\begin{array}{c}\nu_{\text {model }} \\
<\nu\end{array}$ & $\begin{array}{c}\nu_{\text {model }} \\
>\nu\end{array}$ & $\begin{array}{c}\nu_{\text {model }} \\
<\nu\end{array}$ \\
\hline harmonic & 0.0 & 0.0 & 47.0 & 47.6 & 8.2 & 236.5 & 17.3 \\
\hline linear & 0.039640 & 0.0 & 19.6 & 24.7 & 18.2 & 106.9 & 44.0 \\
\hline polynomial & 0.011214 & 0.000010982 & 9.9 & 12.9 & 8.1 & 82.5 & 28.0 \\
\hline quadratic & 0.0 & 0.00001215 & 13.3 & 15.0 & 3.7 & 106.6 & 19.4 \\
\hline
\end{tabular}

Table 2: Mean and maximum errors in DFT harmonic frequencies $\left(\Delta_{\text {harm }}\right)$, excluding excited states and molecules containing atoms larger than $\mathrm{Kr}$. All values reported in units of $\mathrm{cm}^{-1} \cdot{ }^{a} 6-31+\mathrm{G}(\mathrm{d}, \mathrm{p})$ or ${ }^{b} 6-311+\mathrm{G}(\mathrm{d}, \mathrm{p})$ or ${ }^{c}$ aug-cc-pVTZ basis used for anions.

\begin{tabular}{|c|c|c|c|c|c|}
\hline \multirow{3}{*}{ Method } & \multicolumn{3}{|c|}{$\left\langle\left|\Delta_{\text {harm }}\right|\right\rangle$} & \multicolumn{2}{|c|}{$\left|\Delta_{\text {harm }}\right|_{\max }$} \\
\hline & all & $\nu_{\mathrm{e}}^{\mathrm{DFT}}$ & $\nu_{\mathrm{e}}^{\mathrm{DFT}}$ & $\nu_{\mathrm{e}}^{\mathrm{DFT}}$ & $\nu_{\mathrm{e}}^{\mathrm{DFT}}$ \\
\hline & & $>\nu_{\mathrm{e}}$ & $<\nu_{\mathrm{e}}$ & $>\nu_{\mathrm{e}}$ & $<\nu_{\mathrm{e}}$ \\
\hline B3LYP $/ 6-31 G(d, p)^{a}$ & 23.4 & 18.4 & 26.3 & 72.0 & 138.3 \\
\hline B3PW91/6-31G(d,p) ${ }^{a}$ & 20.0 & 19.9 & 20.2 & 102.5 & 94.1 \\
\hline PBE0/6-311G $(\mathrm{d}, \mathrm{p})^{b}$ & 22.2 & 25.2 & 19.2 & 132.9 & 90.4 \\
\hline $\mathrm{M} 05 / 6-311+\mathrm{G}(2 \mathrm{df}, 2 \mathrm{p})$ & 30.4 & 34.5 & 27.6 & 162.3 & 125.7 \\
\hline $\mathrm{M} 06 / 6-311+\mathrm{G}(2 \mathrm{df}, 2 \mathrm{p})$ & 27.5 & 32.5 & 23.7 & 146.4 & 121.2 \\
\hline EDF2/cc-pVTZc ${ }^{c}$ & 21.1 & 21.3 & 21.1 & 76.1 & 82.9 \\
\hline
\end{tabular}

Table 3: Mean and maximum errors in anharmonic frequencies $\left(\Delta_{\mathrm{res}}^{\text {quad }}\right)$ predicted from DFT harmonic frequencies using the quadratic correction model (equation $6, c_{1}=0, c_{2}$ $=0.00001215$ ), excluding excited states and molecules containing atoms larger than Kr. All values reported in units of $\mathrm{cm}^{-1}{ }^{a}{ }^{6} 6-31+\mathrm{G}(\mathrm{d}, \mathrm{p})$ or ${ }^{b} 6-311+\mathrm{G}(\mathrm{d}, \mathrm{p})$ or ${ }^{c}$ aug-cc-pVTZ basis used for anions.

\begin{tabular}{|c|c|c|c|c|c|}
\hline \multirow[b]{2}{*}{ Method } & \multicolumn{3}{|c|}{$\left\langle\left|\Delta_{\text {res }}^{\text {quad }}\right|\right\rangle$} & \multicolumn{2}{|c|}{$\left|\Delta_{\text {res }}^{\text {quad }}\right|$} \\
\hline & all & $\begin{array}{c}\nu_{\text {model }} \\
>\nu\end{array}$ & $\begin{array}{c}\nu_{\text {model }} \\
<\nu\end{array}$ & $\begin{array}{c}\nu_{\text {model }} \\
>\nu\end{array}$ & $\begin{array}{c}\nu_{\text {model }} \\
<\nu\end{array}$ \\
\hline $\mathrm{B} 3 \mathrm{LYP} / 6-31 \mathrm{G}(\mathrm{d}, \mathrm{p})^{a}$ & 23.1 & 23.1 & 23.0 & 123.18 & 133.9 \\
\hline B3PW91/6-31G $(\mathrm{d}, \mathrm{p})^{a}$ & 23.9 & 26.5 & 18.5 & 115.5 & 91.0 \\
\hline $\mathrm{PBE} 0 / 6-311 \mathrm{G}(\mathrm{d}, \mathrm{p})^{b}$ & 26.5 & 31.6 & 16.4 & 133.4 & 87.4 \\
\hline $\mathrm{M} 05 / 6-311+\mathrm{G}(2 \mathrm{df}, 2 \mathrm{p})$ & 32.5 & 39.2 & 18.4 & 161.7 & 102.0 \\
\hline $\mathrm{M} 06 / 6-311+\mathrm{G}(2 \mathrm{df}, 2 \mathrm{p})$ & 29.3 & 34.7 & 16.4 & 152.4 & 100.3 \\
\hline EDF2/cc-pVTZ ${ }^{c}$ & 19.8 & 23.8 & 16.0 & 91.3 & 71.7 \\
\hline
\end{tabular}




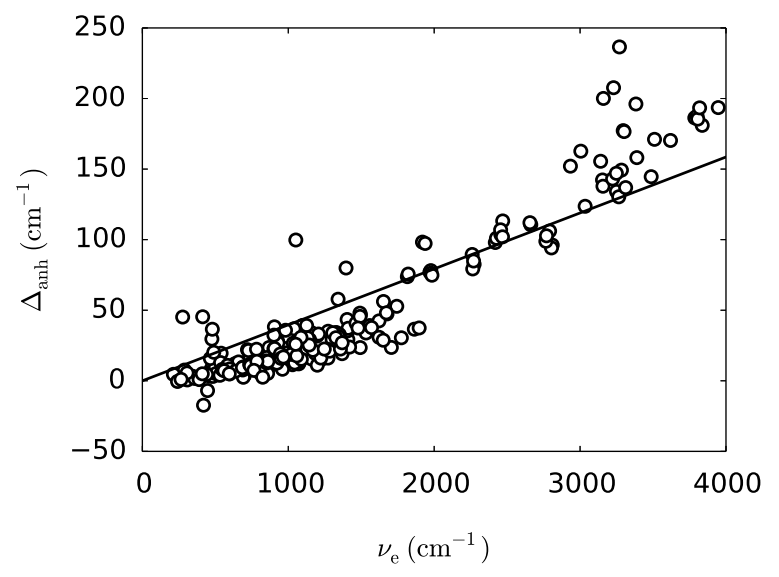

(a)

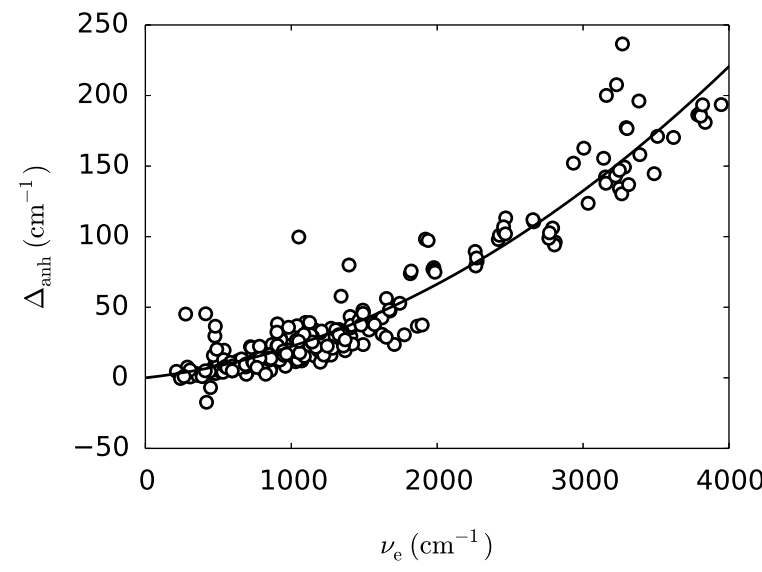

(c)

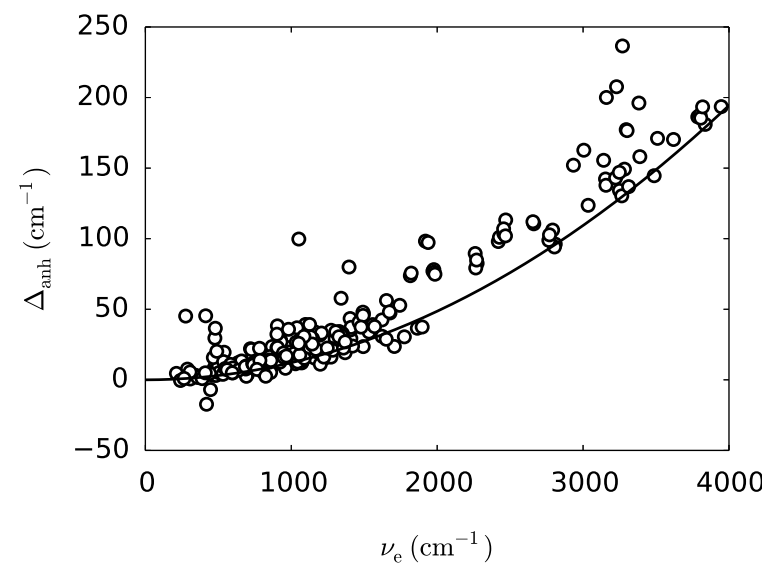

(e)

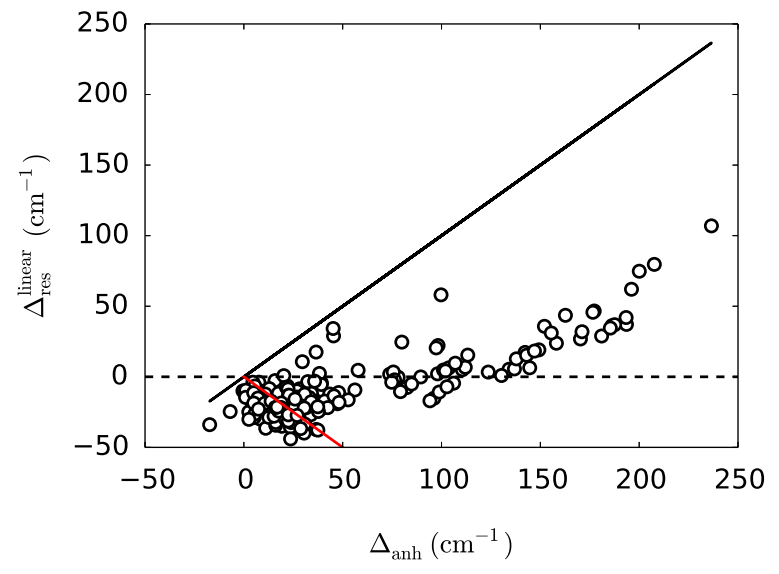

(b)

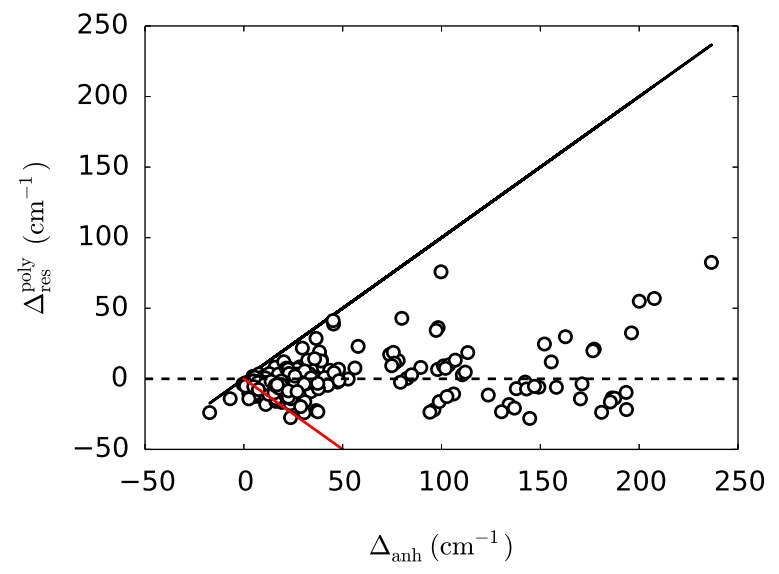

(d)

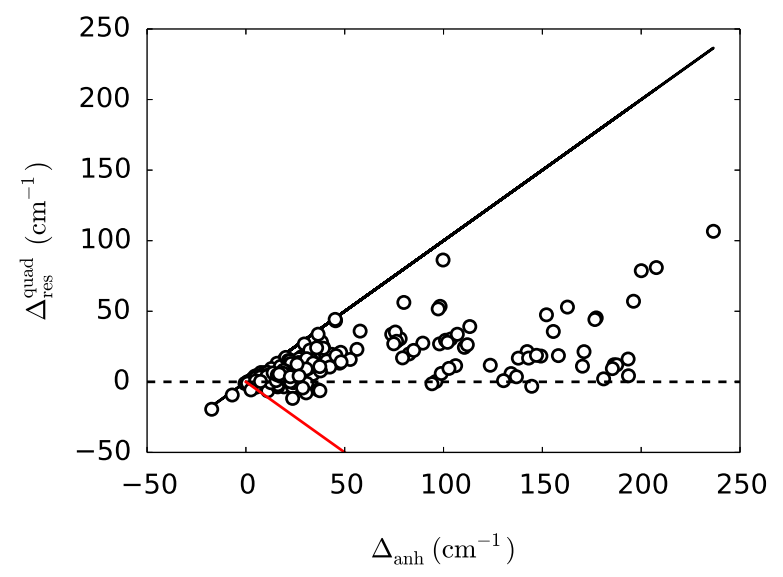

(f)

Figure 1: Left: Benchmark anharmonicities as a function of frequency, with trendlines representing; a) single-parameter linear model, c) dual-parameter polynomial model, and e) single-parameter quadratic model. Right: Corresponding residual errors as a function of anharmonicity, for b) linear, d) polynomial and f) quadratic correction models. 


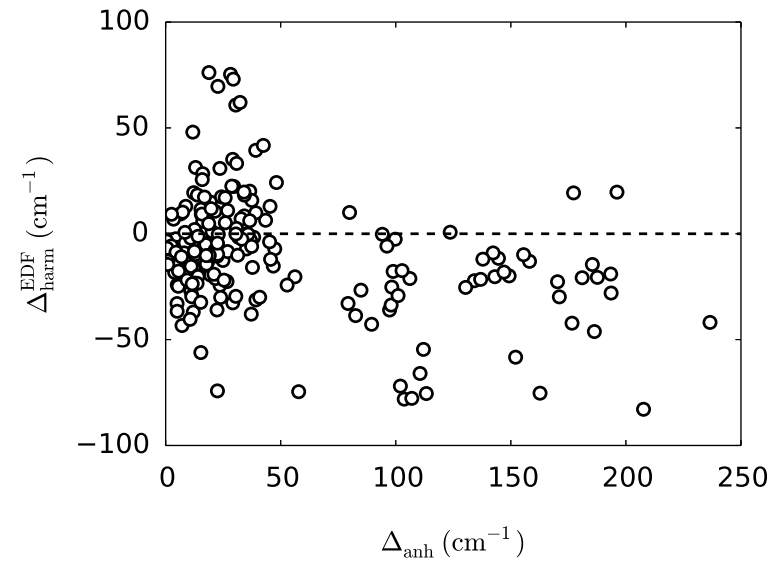

(a)

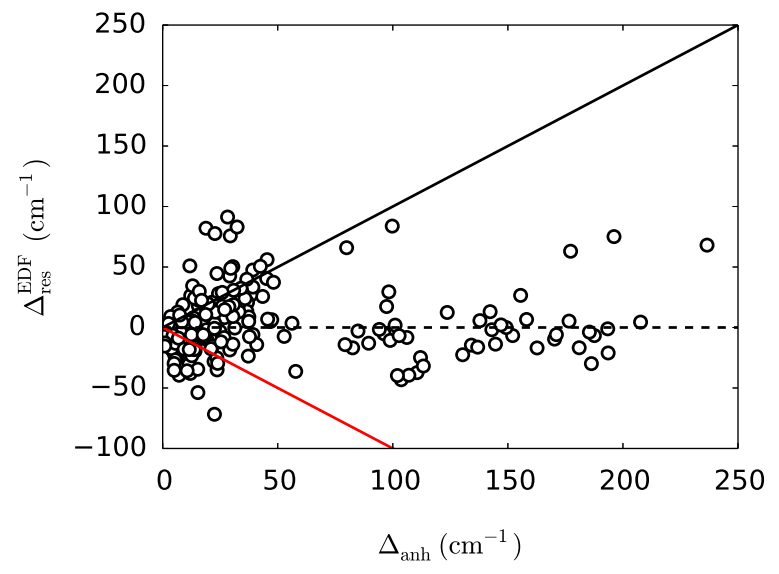

(b)

Figure 2: (a) Errors in EDF/cc-pVTZ harmonic frequencies as a function of benchmark anharmonicity, and (b) corresponding errors in predicted anharmonic frequencies using the quadratic correction model. 


\section{References}

(1) Andersson, M. P.; Uvdal, P. New scale factors for harmonic vibrational frequencies using the B3LYP density functional method with the triple-xi basis set 6311+G(d,p). J. Phys. Chem. A 2005, 109, 2937-2941.

(2) Andrade, S. G.; Goncalves, L. C. S.; Jorge, F. E. Scaling factors for fundamental vibrational frequencies and zero-point energies obtained from HF, MP2, and DFT/DZP and UP harmonic frequencies. J. Mol. Struct. (THEOCHEM) 2008, $864,20-25$.

(3) Laury, M. L.; Boesch, S. E.; Haken, I.; Sinha, P.; Wheeler, R. A.; Wilson, A. K. Harmonic vibrational frequencies: scale factors for pure, hybrid, hybrid meta, and double-hybrid functionals in conjunction with correlation consistent basis sets. $J$. Comp. Chem. 2011, 32, 2339-2347.

(4) Pople, J. A.; Scott, A. P.; Wong, M. W.; Radom, L. Scaling factors for obtaining fundamental vibrational frequencies and zero-point energies from $\mathrm{HF} / 6-31 \mathrm{G}^{*}$ and MP2/6-31G* harmonic frequencies. Isr. J. Chem. 1993, 33, 345-350.

(5) Scott, A. P.; Radom, L. Harmonic vibrational frequencies: An evaluation of HartreeFock, Moller-Plesset, quadratic configuration interaction, density functional theory, and semiempirical scale factors. J. Phys. Chem. 1996, 100, 16502-16513.

(6) Merrick, J. P.; Moran, D.; Radom, L. An evaluation of harmonic vibrational frequency scale factors. J. Phys. Chem. A 2007, 111, 11683-11700.

(7) Sinha, P.; Boesch, S. E.; Gu, C. M.; Wheeler, R. A.; Wilson, A. K. Harmonic vibrational frequencies: Scaling factors for HF, B3LYP, and MP2 methods in combination with correlation consistent basis sets. J. Phys. Chem. A 2004, 108, 9213-9217.

(8) Baker, J.; Jarzecki, A. A.; Pulay, P. Direct scaling of primitive valence force constants: an alternative approach to scaled quantum mechanical force fields. J. Phys. Chem. A 1998, 102, 1412-1424. 
(9) Sibaev, M.; Crittenden, D. L. The PyPES library of high quality semi-global potential energy surfaces. J. Comp. Chem. 2015, 36, 2200-2207.

(10) Coon, J. B.; Cesani, F. A.; Loyd, C. M. Evidence for a double-minimum potential in an excited state of ClO2. Discuss. Faraday Soc. 1963, 35, 118-123.

(11) Peterson, K. A. Accurate ab initio near-equilibrium potential energy and dipole moment functions of the X2B1 and first excited 2A2 electronic states of $\mathrm{OClO}$ and OBrO. J. Chem. Phys. 1998, 109, 8864-8875.

(12) Lin, C. Y.; George, M. W.; Gill, P. M. W. EDF2: A density functional for predicting molecular vibrational frequencies. Aust. J. Chem. 2004, 57, 365-370.

(13) Stephens, P. J.; Devlin, F. J.; Chabalowski, C. F.; Frisch, M. J. Ab initio calculation of vibrational absorption and circular-dichroism spectra using density-functional force fields. J. Phys. Chem. 1994, 98, 11623-11627.

(14) Becke, A. D. Density-functional thermochemistry .3. The role of exact exchange. J. Chem. Phys. 1993, 98, 5648-5652.

(15) Adamo, C.; Barone, V. Toward reliable density functional methods without adjustable parameters: The PBE0 model. J. Chem. Phys. 1999, 110, 6158-6170.

(16) Zhao, Y.; Schultz, N. E.; Truhlar, D. G. Exchange-correlation functional with broad accuracy for metallic and nonmetallic compounds, kinetics, and noncovalent interactions. J. Chem. Phys. 2005, 123, 161103.

(17) Zhao, Y.; Truhlar, D. G. The M06 suite of density functionals for main group thermochemistry, thermochemical kinetics, noncovalent interactions, excited states, and transition elements: two new functionals and systematic testing of four M06-class functionals and 12 other functionals. Theor. Chem. Acc. 2008, 120, 215-241.

(18) Curtiss, L. A.; Raghavachari, K.; Redfern, P. C.; Rassolov, V. A.; Pople, J. A. Gaussian-3 (G3) theory for molecules containing first and second-row atoms. $J$. Chem. Phys. 1998, 109, 7764-7776. 
(19) Frisch, M. J.; Pople, J. A.; Binkley, J. S. Self-consistent molecular-orbital methods .25. Supplementary functions for Gaussian basis sets. J. Chem. Phys. 1984, 80, $3265-3269$.

(20) Krishnan, R.; Binkley, J. S.; Seeger, R.; Pople, J. A. Self-consistent molecularorbital methods .20. basis set for correlated wave-functions. J. Chem. Phys. 1980, 72, 650-654.

(21) Dunning, T. H. Gaussian basis sets for use in correlated molecular calculations .1. The atoms boron through neon and hydrogen. J. Chem. Phys. 1989, 90, 1007-1023.

(22) Woon, D. E.; Dunning, T. H. Gaussian basis sets for use in correlated molecular calculations .3. The atoms aluminum through argon. J. Chem. Phys. 1993, 98, $1358-1371$.

(23) Clark, T.; Chandrasekhar, J.; Spitznagel, G. W.; Schleyer, P. V. R. Efficient diffuse function-augmented basis sets for anion calculations. III. The $3-21+\mathrm{G}$ basis set for first-row elements, Li-F. J. Comp. Chem. 1983, 4, 294-301.

(24) Kendall, R. A.; Dunning, T. H.; Harrison, R. J. Electron affinities of the first-row atoms revisited. Systematic basis sets and wave functions. J. Chem. Phys. 1992, 96, 6796-6806.

(25) LaJohn, L. A.; Christiansen, P. A.; Ross, R. B.; Atashroo, T.; Ermler, W. C. Ab initio relativistic effective potentials with spin-orbit operators .3. Rb through Xe. $J$. Chem. Phys. 1987, 87, 2812-2824.

(26) Ross, R. B.; Powers, J. M.; Atashroo, T.; Ermler, W. C.; LaJohn, L. A.; Christiansen, P. A. Ab initio relativistic effective potentials with spin-orbit operators .4. Cs through Rn. J. Chem. Phys. 1990, 93, 6654-6670.

(27) Shao, Y. et al. Advances in molecular quantum chemistry contained in the Q-Chem 4 program package. Mol. Phys. 2015, 113, 184-215. 
(28) Biczysko, M.; Panek, P.; Scalmani, G.; Bloino, J.; Barone, V. Harmonic and anharmonic vibrational frequency calculations with the double-hybrid B3PLYP method: analytic second derivatives and benchmark studies. J. Chem. Theor. Comp. 2010, $6,2115-2125$.

(29) Boese, A. D.; Klopper, W.; Martin, J. M. L. Anharmonic force fields and thermodynamic functions using density functional theory. Mol. Phys. 2005, 103, 863-876. 
ToC graphic

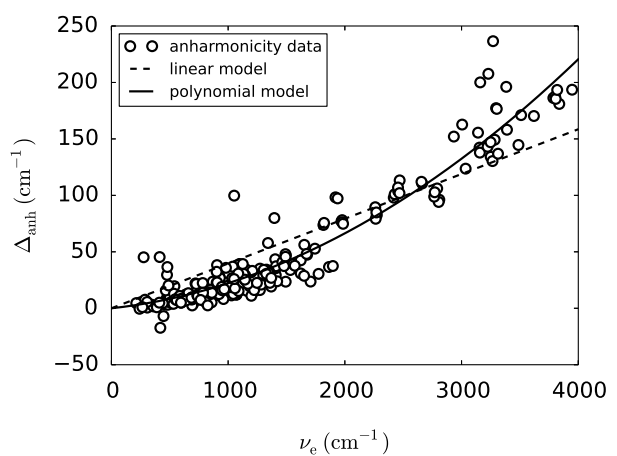

